\title{
LE SESTE DEL LAGO DI SANTA CROCE
}

\author{
Domenico Di Rimpro
}

1. - Sllo soppo di porlare un contribulo allo sludio si-lemalieo delle catratteristiche fisiehe dei laghi ilaliani intrapreso dall'Istitule Vizionale di Geofi-iral. ho escruilo il ealcolo delle sesse del lagro di Sillla Cirore (Bellumo).

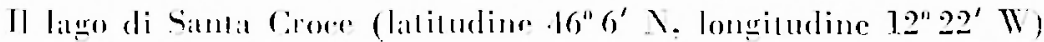
ron if lange Morlo. nelle Prealpi venele, vengeno rhiamali lapisini dalla vieina regione dell Alpago ed ocelepano parte del fondo della profonda incianaluta, chiantala anch'essa valle lapisina, che separa

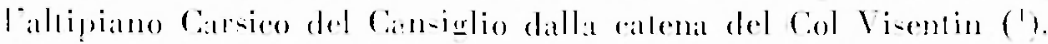

Il terribile lerremolo del 1873, il ani cpicentro corrispose alppluno alla regiome dell Nlpago. alliro l'allenzione di illustri geologri -u que-li luoghi. i quali si orcuparono anche dellorigrine di questi laghi. problema di un erero inlereses dal punlo di vi=la geolog̣ieo. rhe ancoral $\dot{e}$ in di-cusione.

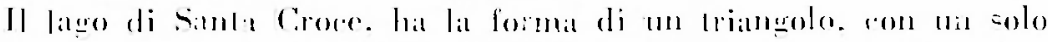

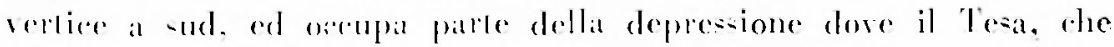

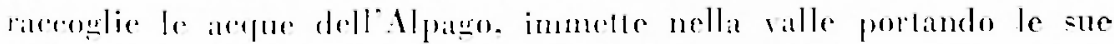

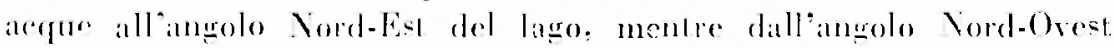
sortomo le acepue del Ria. affluente del Pialve.

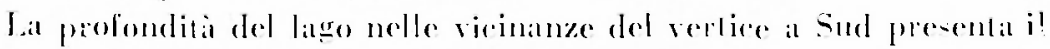
masimo valore di rirrat $35 \mathrm{~m}$ e va poi man mano diminuende verso la base a Nord. Il fomdale abhastanza regolate si presenta pero pieno di

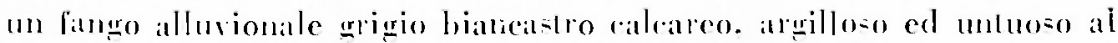
latlo. A Sud del liago la valle si restringe ed a orerupala dalle famose franc di Fadalto, il rui punlo più depresso is alıo m 482.

I principali dali norfonetriei del lago di Santa Croce sono:

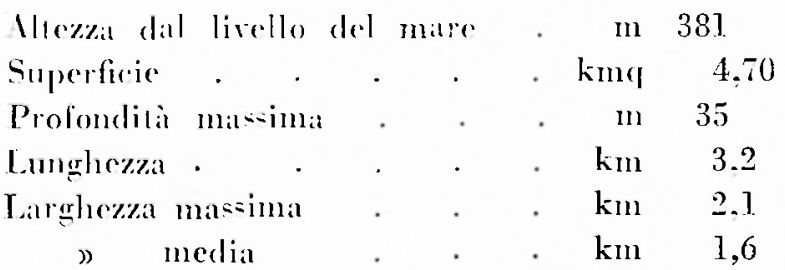




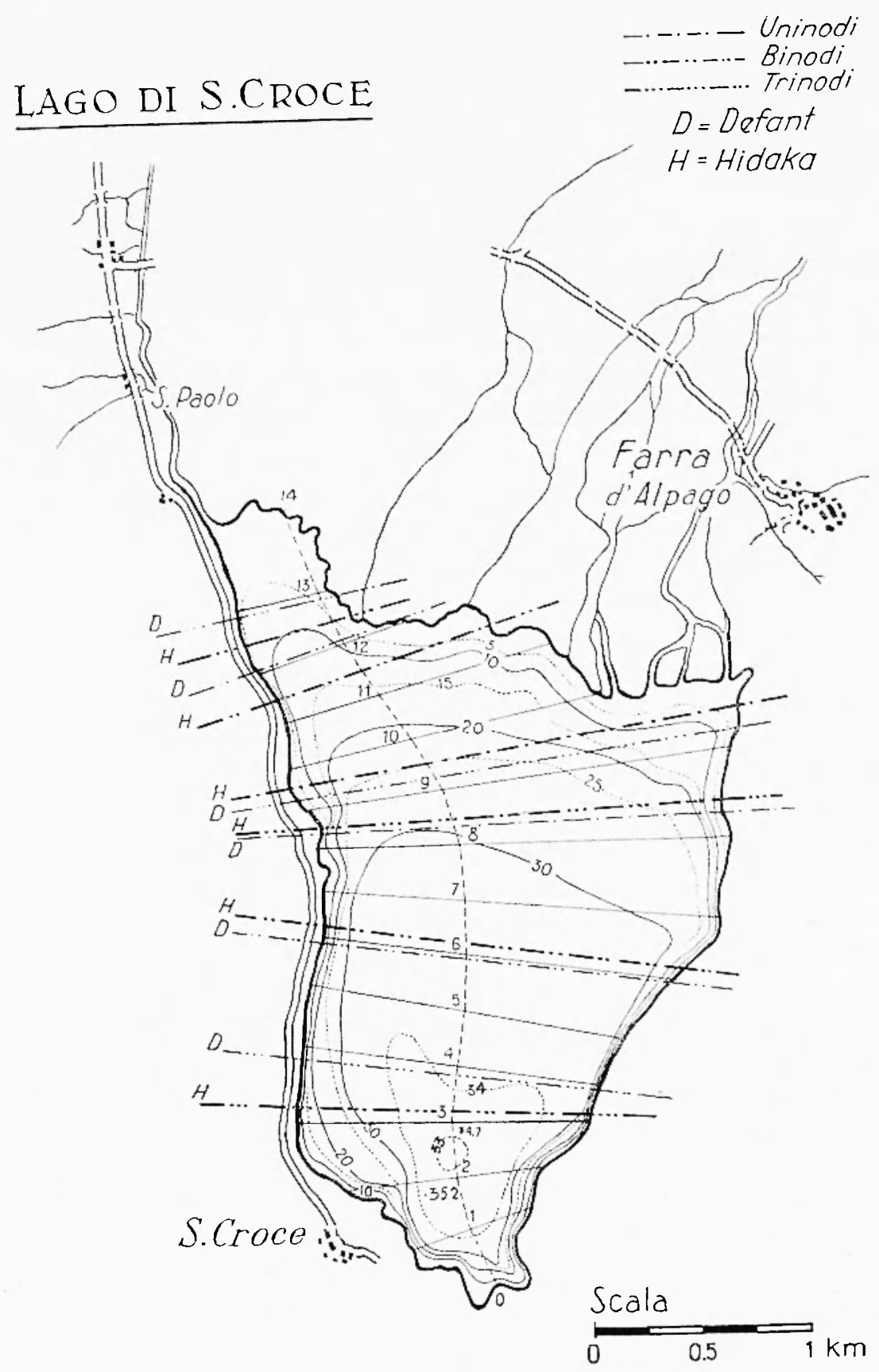


2. - Partendo dallequazione fondamentale della idrodinamica, Chrystal, considerando nu lago come un canale funilo di sezione rariabile con la profondila non in modo bruseo, Iralla in maniera rigorosal la prima reoria per lo sludio delle sesese.

Chrystal consirlera tracurabili lo componenti laterali del movimento orizontale in modo da tener conto solo del movimento nel senoo dell'ase $x$, posto lungo la linea di valle e allal superficie del lag̣o; piecolo lo -po-limento 1 reslieale li-pello alla profondià he dipendente erelusivamente dall'ascissal $x$ e dal tempo $t$, in modo da risulate uguale per tute le particelle che si frovano in un dalo istante in ma sezione verticale $S(x)$ del lago, normale alla linea di valle; infine. per le onde di lungherza lale per eni la profondia $h$ del barino sia piecola, mascurabile l'aceelerazione verticale, da rui

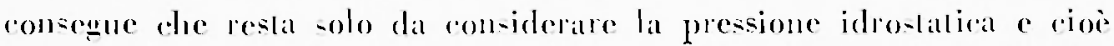
solo le variazioni di presione derivanti dalle rariazioni di livello.

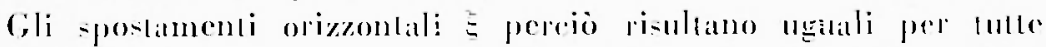
le parlicelle di una -lesil sezione $S(x)$. Ciò poslo Chry-lal perviene alla seguente expluzione, rhe portal il suo nome:

$$
\sigma(v) \frac{d^{2} u}{d v^{2}}+\frac{4 \pi^{2}}{T z} u=0
$$

dove u rappresenta it volume del liquido che allrarersa las sexione $\rightarrow(x)$ in forza dello postamento orizzontale $\equiv$ e v l'arcal. calcolala -ulla -uperfirie libera da ma estremila del lago, $x=0$, alla sezione

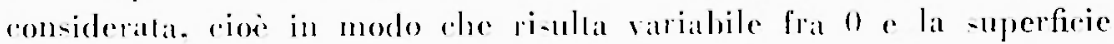
loliale del laigo a.

lat fumpione $\sigma$ e dala dal prodollo dellatrea $S(x)$ di mat -ezione lasserale per lat larghezzal in superfiede $b(x)$ della sessa sezione del !aigo.

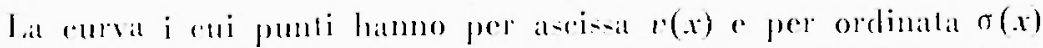
fu chiamata da Chry-tal "rumal dormalen e rappresenta la sezione longitudinale lungero la linea di valle del laggo ridollo.

La "rurval normale " del lago di Sanla Croce, come lulli wh altri elementi. tho delerminialat servendomi dei dali ollenuli da ma calla balimetria al 25000 rilerata dal Magrini. in mal campagna dellautumo del 190)t, ron 34.3 scandagli a con le rurve di profonditi tracedate di 5 in 5 m ollere ad allri numeros punti segnati con profondilà intermedic. 
Su di essal, con la malssima preci-ione, lo tratceialo 13 sezioni arl mal distanza l'ma dall'allra in generale di 250 m rireal, normali alla linea di valle, che partendo dal vertice Sud del lago (abilato di Santa Croces) seguendo la linea di massima profondila e lenendo comlo della lorma particolare del haleino, arriva alla parle Nord-Orest della base.

Nella labella I riporlo i valori delle errandeze $\left[x_{3} S(x), b(x)\right.$, $f(x)$. $\sigma(x)]$ nocesilri per i calcoli steressivi.

Lal fegura l rapperenenta la rurva normale reles, pur non present-

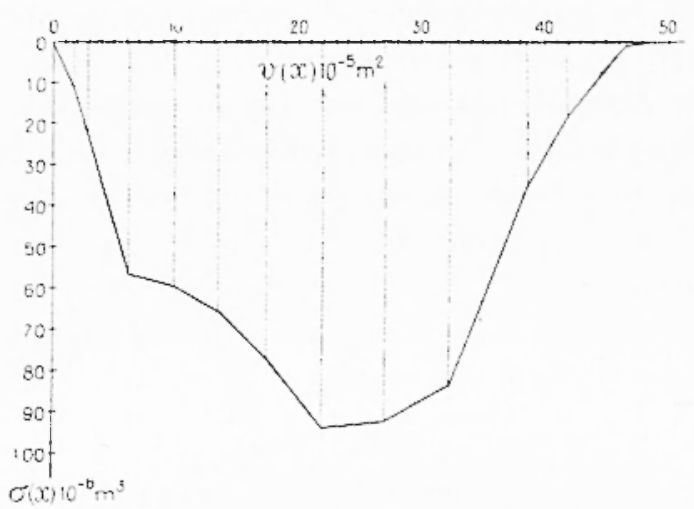

Fig. 1 landosi execsivamento irregolare, $101 \mathrm{l} a \mathrm{vi}$ non is fareilmente rat)presentiblile dil umit -emplier fumzione analiliea e pertanto nom iv posibile inferrare la repuazione di Chrystal rom i motodi da lui stweriti.

Per pue-to -ludia mi sono vilto dei melodi di Midakil e ali Defant che riescono più agevoli deoli altri e i cui ri-nhali sono egualnu, ule buoni.

3. - Welodo di Mirkala: Applienudo il calcolo delle rariazioni del Ril\% all’inlewrazione dellepuzione differenziale di Chryslal. Thidaka ha proposto un metorlo piullosto semplier per deleminare

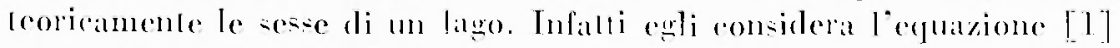
ron le rondizioni ai limiti

$$
u(o)=u(a)=0
$$

- poso $z=\frac{v}{a}$ la laisforma nolla

$$
\frac{d^{2} u}{d z^{2}}+\frac{i}{\sigma(z)} u=0,
$$

dove

con le condizioni al Jimili

$$
\hat{\lambda}=\frac{1 \cdot \pi^{2}}{T:} \frac{a^{\prime \prime}}{g},
$$

$$
u(o)=u(1)=0 \text {. }
$$




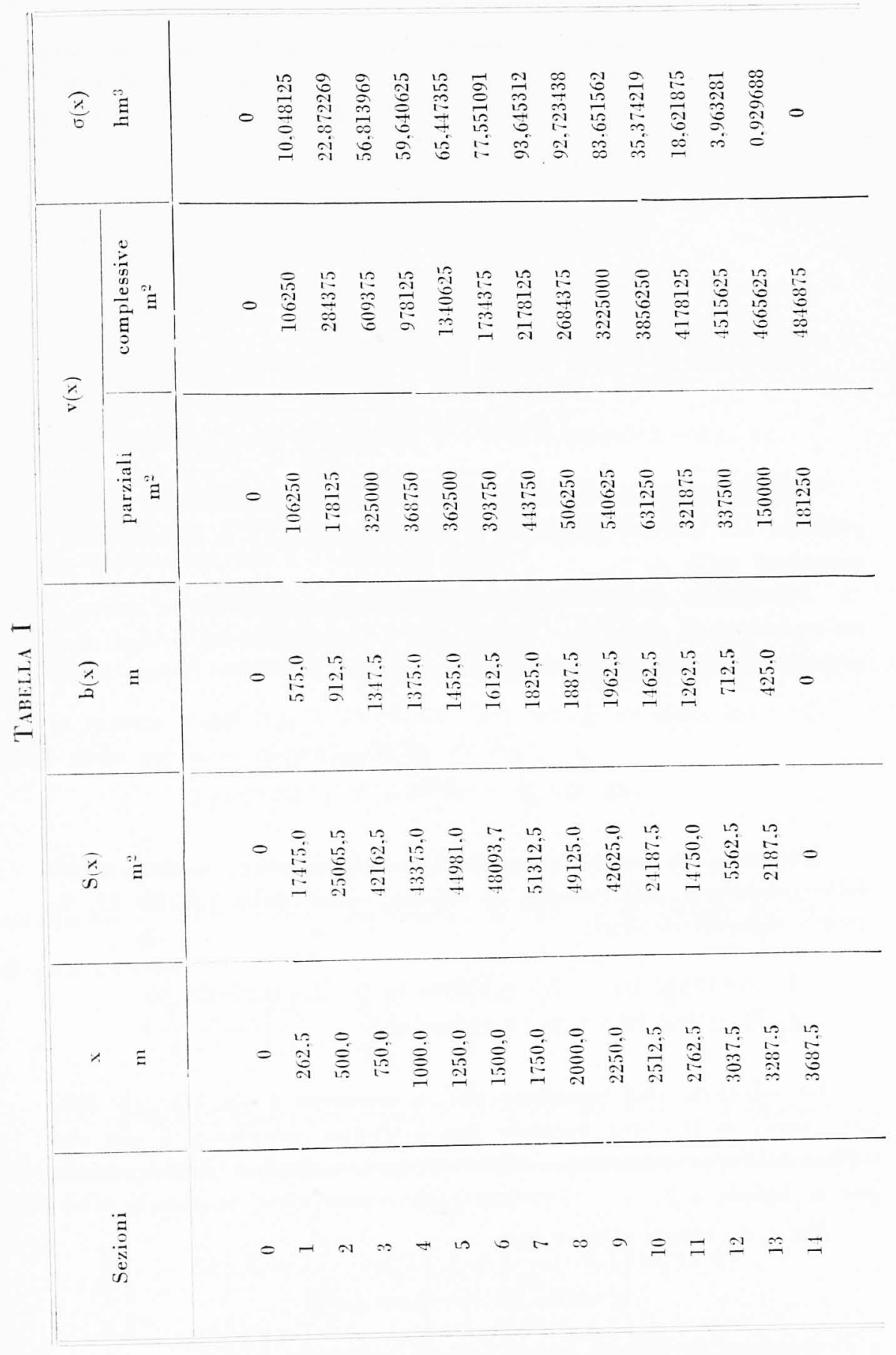


L.t [ㄹ], alle condizioni posle, risulta soddiefilla solo da particolari valori di h. Ilidaka, applicando un noto leorema del calcolo delle variazioni, mo-lra rhe l’integrazione dellequazione [2] equivale alla ricerea del minimo valore dell'inlewrale:

$$
I(u)=\int_{i}^{1}\left\{\left(\frac{d u}{d z}\right)^{2}-\frac{\lambda}{\sigma(z)} u^{2}\right\} d z
$$

e propone per a l'espresione:

$$
u=\stackrel{\Delta}{0}_{i}^{m}-A_{i} z(1+z)
$$

Derivando fuesta efuazione rispello a $=0$ sosituendo la derivala ollenula rol relativo valore di $u$ aell’integrale $[3]$ i hamo ( $m+1)$ repuazioni nella $t$.

Ammulando il determinanle dei coeffeienti delle $f_{i}$-i atriva al

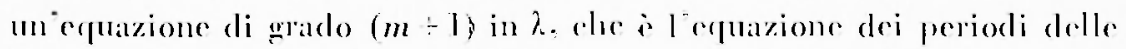
sese ricercile. In questal equazione:

$$
I_{\mathrm{n}}=\int_{\mathrm{i}}^{1} \frac{z^{2}(1-z)^{2} z^{\mathrm{n}}}{\sigma(z)} d z .
$$

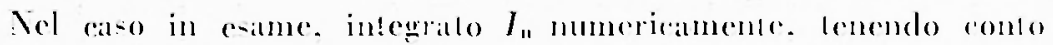
delle rondizioni agli estremi, il alcolo, iome dalla laledla Il. hal lallo i -ceguenti risulati:

$$
\begin{aligned}
& I_{11}=0.611352 \quad 10^{-*} \quad I_{1}=0.378996 \quad 10^{:} \quad I_{2}=0.27 .517910^{-*} \\
& I_{3}=0.217762 .10^{-3} \quad I_{1}=0.180602110^{-3}
\end{aligned}
$$

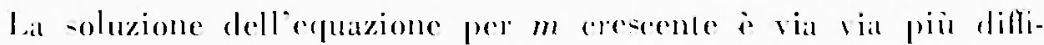

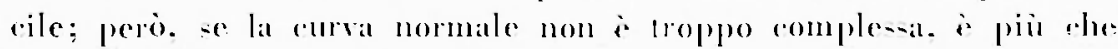
suffeiente l'approsimazione ollemula con le soluzioni dell'epuatione pere m ungule a 2.

Pre u in rue-to caro -i ha:

$$
u=z(1-z)\left(A_{1}+A_{1} z+A_{2} z^{2}\right),
$$

e leepuazione di Ilidaka per i periodi, -riluppalal, ri-ulaa: 


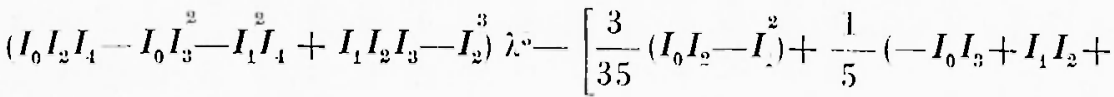

$$
\begin{aligned}
& \left.\left.+I_{1} I_{3}\right)+\frac{2}{15} I_{0} I_{4}+\frac{1}{3}\left(-I_{1} I_{4}-\stackrel{2}{I_{2}^{2}}+I_{2} I_{3}+I_{2} I_{4}-I_{3}\right)\right] i^{2}+\left(\frac{1}{700} I_{0}-\right. \\
& \left.-\frac{3}{350} I_{1}+\frac{53}{2100} I_{2}-\frac{1}{30} I_{2}+\frac{1}{60} I_{i}\right),-\frac{1}{10500}=0,
\end{aligned}
$$

le rui radici permettono di determinare $\mathrm{j}$ periodi delle tre prime sesse.

So-tituendo in rue-a erguizione $\mathrm{i}$ cinrue valori di I calcolali.

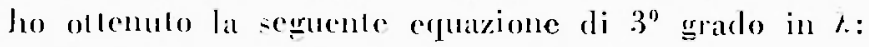

$$
10^{-9} 0.52522^{3}-10^{-6} 2.8246057^{2}+10^{-3} 3,4.54395 \% 1 .-1=0 \text {, }
$$

che risolta col melodo di Newton, per cueressive approsimazioni. partendo dai due valori di \% rieavali dallecquazione di Midiaka per $m=1$. mi hat dito per $\lambda$ i ecouenti valori:

$$
\lambda_{1}=126.29, \quad \lambda_{.2}=1183 ., 06, \lambda_{.3}=3768.21 \text {. }
$$

Que-1i valori -0-tiluiti nell'espressione $1=\frac{4 \pi^{2} a^{2}}{T^{2} g}$, Ienendo conlo che la -uperfieie del lago is a-48-1687is m². mi hamno dalo per i periodi delle tre -ane rispetlisamente $i$ valori:

$$
T_{1}=7^{m}, 84 \quad ; \quad T_{z}=1^{m}{ }_{2} 70 ; \quad T_{3}=2^{m}, 64 .
$$

Liner nodeli. -.- Per la determinazione delle linee nodali vale la condizione $\frac{d u}{d z}=0$, pereio derivando la $[.5]$ e dividendo per i puis serivere:

$$
4 \frac{A_{2}}{A_{0}} z^{3}+3\left(\frac{A_{1}}{A_{0}}-\frac{A_{2}}{A_{0}}\right) z^{2}+2\left(1-\frac{A_{1}}{A_{0}}\right) z-1=0 .
$$

Per ollenere i valori dei rapporti $\frac{A_{2}}{A_{0}}$ e $\frac{A_{2}}{A_{0}}$ per i tre tipi di sese ronsiderati, ha-lat risolvere il seguente -i-lema, tenendo conto dei valori delle grandeza precedentemente calcolate:

$$
\begin{aligned}
& \left(\frac{1}{3}-I_{0} \lambda_{2}\right) A_{1}+\left(\frac{1}{6}-I_{2} \lambda\right) A_{i}+\left(\frac{1}{10}-I_{2} \lambda\right) A_{2}=0 \\
& \left(\frac{1}{6}-I_{1} \lambda\right) A_{0}+\left(\frac{2}{15}-I_{2} \lambda\right) A_{1}+\left(\frac{1}{10}-I_{3} \lambda\right) A_{2}=0
\end{aligned}
$$




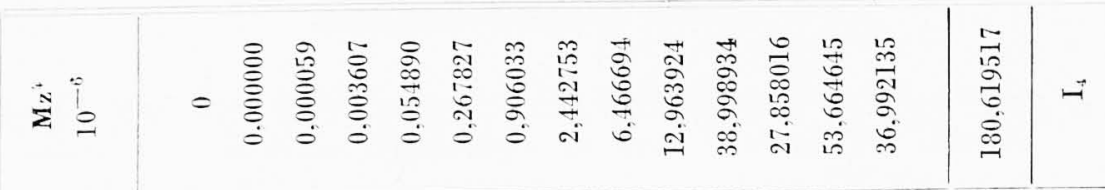

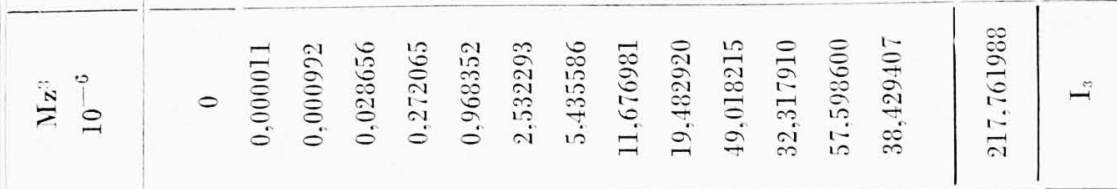

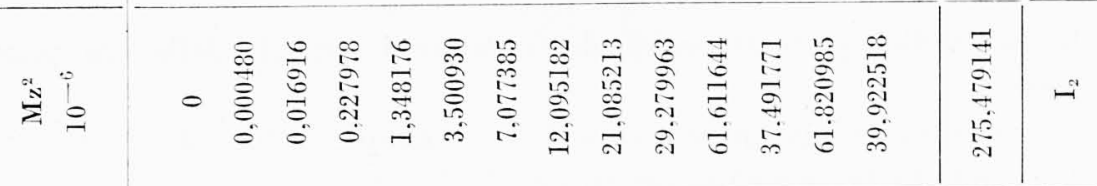

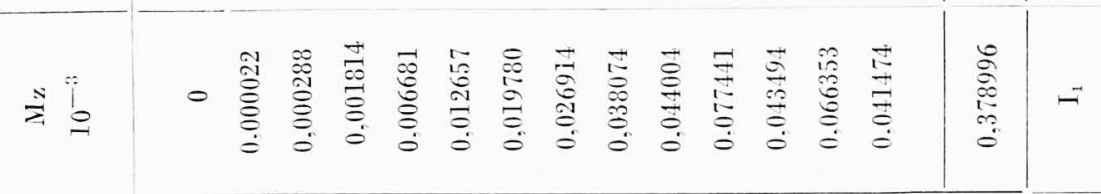

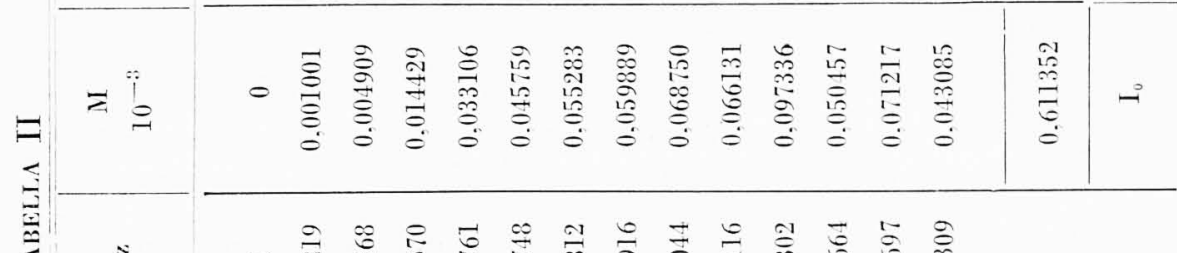

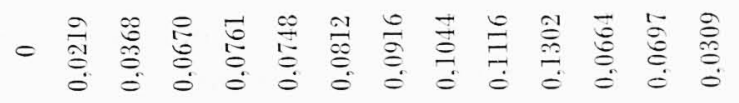

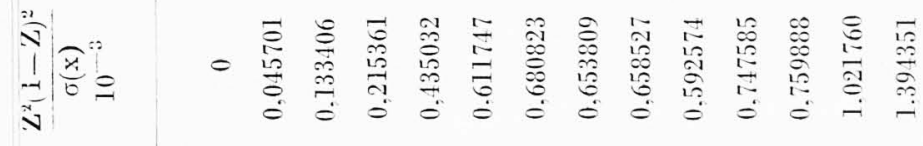

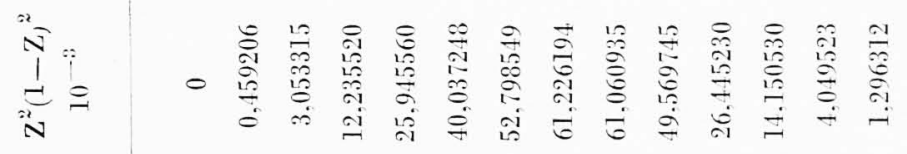

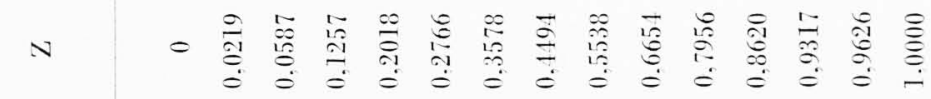

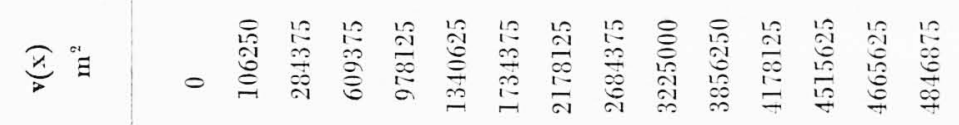

$$
\begin{aligned}
& \text { !uotzes } \mid=-a \text { a }
\end{aligned}
$$


Per $\lambda_{1}=426: 29$ sono risulati per la sessa minodale $i$ valori dei rapperrti:

$$
\frac{A_{1}}{A_{0}}=-1,947 \quad \frac{A_{10}}{A_{0}}=3,601 .
$$

liequazione [6] diviene percio:

$$
14,104 z^{3}-16,641 z^{-}+5,891 z-1=0
$$

la rui unica radice arectabile, perchè minore dell'unilà, è:

$$
z_{1}=0,1218 \text {. }
$$

Liminodo viene perció a cadere tra la $9^{a}$ e la $10^{2}$ sezione, a km 2.364 dill'origine po-li, come precedentemente delto, nel verlice Sud del lago.

Suecesivamente, per la binodale $l_{2.2}=118.5,66$, rimblano

$$
\frac{A_{1}}{A_{0}}=2,323 \quad-\quad \frac{A_{2}}{A_{0}}=-4,626
$$

- I'mplizione dei nodi cliviene

$$
18,504 z^{3}-20,847 z^{2}+2,646 z+1=0,
$$

le rui due solurioni arceltilbili =ono

$$
\left.z_{1}=0.3820\right) \quad z,-0.9015 \text {. }
$$

I due nodi relalivi ditaino dall origine km 1,5.5.5 c 2,919.

Inalogamente, per la trinodale $\lambda_{3}=3768,21$ e i rapporti risul. limo:

$$
\frac{A_{1}}{A_{0}}=-3.922 .2 \quad \frac{A_{2}}{A_{0}}=3,1739 .
$$

Liequazione nelle $z$ risula:

$$
12,6956 z-21.2883 z^{2}+9,844-4 z-1=0
$$

Ir cui colurioni -ono:

$$
z_{1}=0.1 \cdot 109 \quad z_{2}=0,5922 \quad z_{3}=0.9434 \text {. }
$$

I nodi -i trovano rispellixamente a $0,300,2,086,3.132 \mathrm{~km}$ dalla origine.

Distribuzione delle ampiezze. - L'andamento delle ampiczae -i deremina ron-iderando la relazione

$$
\eta=-\frac{d u}{d v}=-a \frac{d u}{d z} .
$$


Sostituendo a $\frac{d u}{d z}$ lat sua cspresione e tenendo conto delle sriandezze ralcolate. -i perviene alle seruenti tre relazioni rispettivanente per le sesse uni-bi-trinodali

$$
\begin{aligned}
& \zeta^{\prime}=\frac{\eta}{a \cdot t_{0}}=-\left(14.101 . z^{3}-16.611 z^{2}+5,89 \cdot 1 z-1\right) . \\
& \zeta^{\prime \prime}=\frac{\eta}{a \cdot t_{n}}=-\left(-18,501.1 z^{3}+20.8 \cdot 17 z^{2}-2,6 \cdot 16 z-1\right) . \\
& \zeta^{\prime \prime \prime}=\frac{\eta}{a \cdot t_{n}}=-\left(12.696 z-21.288 z^{2}+9.411 z-1\right) .
\end{aligned}
$$

Dando a z suecesivamente $i$ valori corri-pondenti ad ogni se-

\begin{tabular}{|c|c|c|c|c|}
\hline \multirow{2}{*}{ 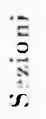 } & \multirow{2}{*}{$x$} & \multicolumn{3}{|c|}{$\zeta$} \\
\hline & & 1 & 11 & 111 \\
\hline 0 & 0 & +1.0000 & -1.0000 & +1.0100 \\
\hline 1 & 262.5 & +0.879 & $-1.018 \%$ & +0.791 \\
\hline$\underline{2}$ & 5010.11 & +0.708 & -1.087 & +0.103 \\
\hline 3 & 7511.0 & +0.193 & $-1,0.10$ & +0.07 .1 \\
\hline 4 & 10000.0 & +0.370 & $-0.83 i$ & -0.22 .1 \\
\hline 5 & 1250.0 & +10.338 & -0.529 & -0.363 \\
\hline 6 & $15(10.0)$ & +0.362 & -0.125 & -0.379 \\
\hline$i$ & 1750.0 & +0.110 .5 & +0.312 & -0.277 \\
\hline 8 & 20000,0 & +0.39 .1 & +10.785 & $-(0.079$ \\
\hline 9 & 2250.0 & $\therefore 0.201$ & +1.018 & +0.135 \\
\hline 10 & 2.512 .5 & -0.408 & +0.772 & +0.2 .19 \\
\hline 11 & 2762.5 & -0.939 & $+0,357$ & +0.201 \\
\hline 12 & 3037.5 & -1.693 & $-0,331$ & +0.0 .10 \\
\hline 13 & 3287.5 & -2.1999 & -0.735 & $-0,071$ \\
\hline 14 & 3687,5 & -2.651 & -1.303 & -0.252 \\
\hline
\end{tabular}
zione. lo calcolati i valori delle anp,ieze che riporto nella tabe!la lll,

TIBELIA III menter nelle figure: 3. 1, i grafici relativi (lines traltergeriate) danno nom idea rliara ed immediata dellandamento delle ampiene per il relativo lipo di sessa.

1. - Meloclo di Defarte Questo metodo prende pure le moese dalle cefuazioni dillerenziali londamentali dellodedinamiea (equazioni del movimento o dellat rome timita) e pone at solito laise $x$ lungo la lima di valle roll'origgine ad un estremo del lago e comidera la massa d'actual rompresa fra due sezioni di spesore dx enue lumale ad $S(x)$, dx.

Se pri stati s spostano orizzontalmente di . verticalmente di 1 , le equazioni del moto e della contimuita sono soddisfatte solo da autoralori di un paramero, che nel caso rappresentano i periodi delle oseillazioni libere. Il Defant integrando le due equazioni pervienc alle -eguenti relazioni: 


$$
\begin{aligned}
& 2 \Delta \eta_{0}=\frac{4 x^{2}}{g T^{2}} 2 \vdots_{0} \Delta x, \\
& 2 \Xi_{0}=-\frac{1}{S(x)} \int_{0}^{\mathrm{x}} 2 \eta_{0} l(x) d x,
\end{aligned}
$$

dove $2 \eta_{0}$ e $25_{0}$ rappresentano le ampiezze delle oseillazioni rertieali e orizontali lungo il layo e gli altri simboli sono ghi tesi preere dememente adoperali.

Quese due relazioni dammo la posibilita di ralleolare contemporatneamente il periodo della se-sal e il relativo andamento dell'ampiezzal per successive approsimazioni, una volta noto un valore approsimato del periodo slewe.

Diliali note le mi-ure delle sezioni s(x) e quelle delle superficie parziali del lago eompreso trat sezione e sezione (libella l) e fissillo uno -po-tamento arbitrario verticale $\eta=100(\mathrm{~m}$ in rorri-pondenzal dellorigine con le condizioni $\Xi(0)=0$, $\xi(l)=0$ alle entemita della linea di valle. is powilite ratrolate il valore delle due esprenioni stl serilte.

Se il numero delle seziond a suf-

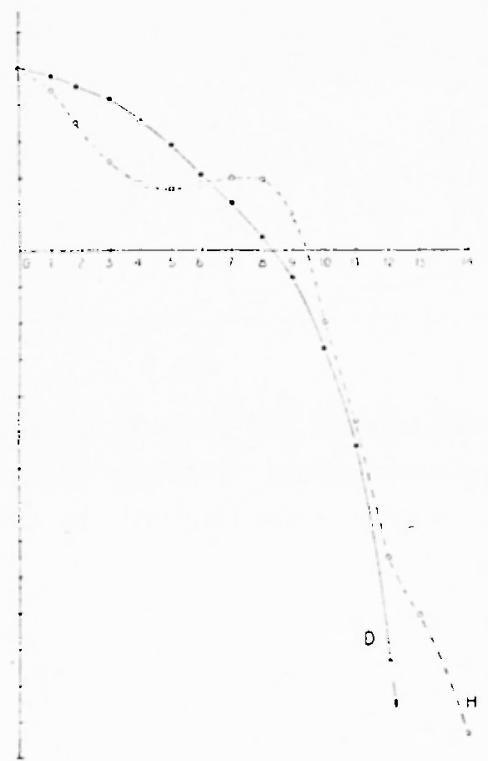

Iï. 2 fieientemente grinde, si può ronsiderare, con buona applos-imazione, che tra loorignine e la prima sezione lo spostamento verticale rimangag costante; allora si può cal-

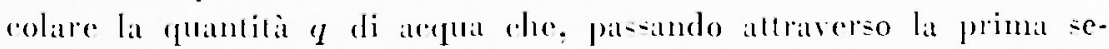
zions, determina lo spostamento:

$$
\eta=\int_{0}^{x_{1}} 22_{0} h(x) d(x)=21_{0} \int_{0}^{x_{1}} h(x) d(x)=2 n_{0} v\left(x_{1}\right)
$$

Otlenuto cosi il valore dell'integrale e nota la $S(x)$ corrispondente, $-i$ determina $25_{0}$ dellat seconda relaxione: sostuito nellat prima permete di calcolare $2 \mathrm{~J}_{0} \mathrm{o}$ di conseguenza il valore dello spostamento verticale in romispondenza della prima sezione, che risulta 
J00+2 $+2 \eta_{0}$. Si ripelono i alleoli per lulle le successive sczioni fino all ultima. considerata di area nulla, in corriepondenza della ruale si dovri anmulare $a$.

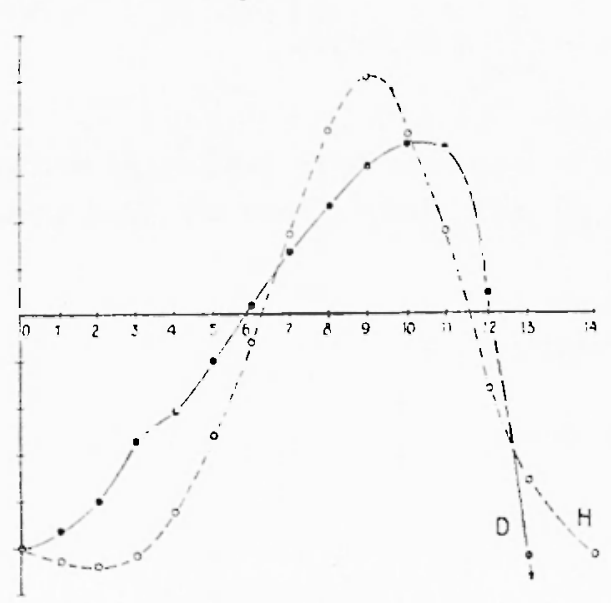

Fig. 3

Se il periodo non roinvide con l'esillo periods della sessa, il raleolo va ripetuto avendo cura di diminuire il periodo precedencomente adoperalo se $q$ i magraiore di 0 per le sesse a nodi dispari; c so $q$ is minore di 0, atumentarlo. Per le sesse a nodi pari hisognal operare in maniora contraria.

I periodi inizialmente adoperali in ruesto raso sono slati ruelli ollenuti preredentemente col melode di Itidaka: i quali, correnti con sueces-ive approssimazioni, enendo conto li volta in volta delle rondixioni su exposte, sono risultali. in definitiva, per i lre lipi di sessa:

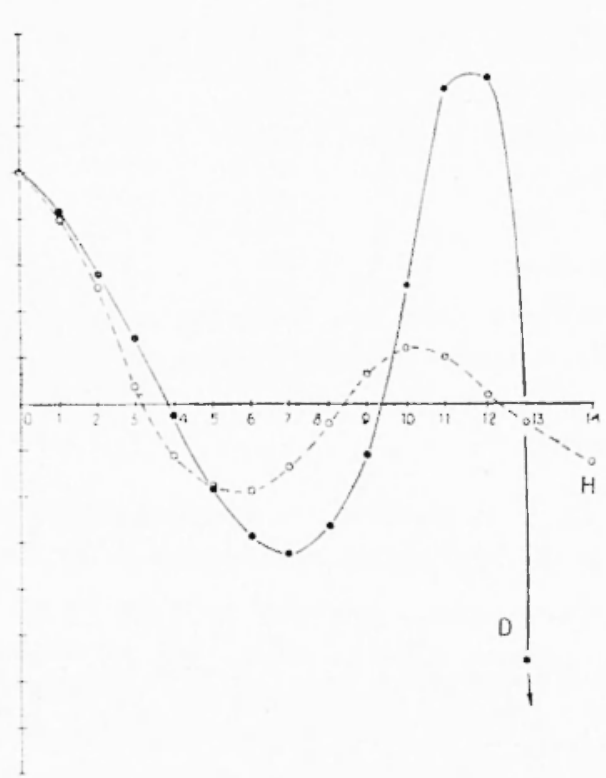

Fig. 4
$T_{1}=7^{m}, 13$ per l'uminodale. $T_{0}=4^{\mathrm{m}} .9$ per la hinodale. $T_{\mathrm{s}}=3^{\mathrm{m}}, 2 \mathrm{2}$ per la trinodiale.

lo sviluppo dei ralcoli riportato nclla tabella IV. dove risultano per le q finali ralori abbastanza pire. roli, ma non nulli; pero lapprosimazione i pii ahe sufferiente per il problema in ceiame.

Questo metodo, como abbiamo erià dello, pormolle contemporancamente alla determinazione del periodo quella dei nodi . l'andamento decrli spostamenti rerlicali nelle sin- 


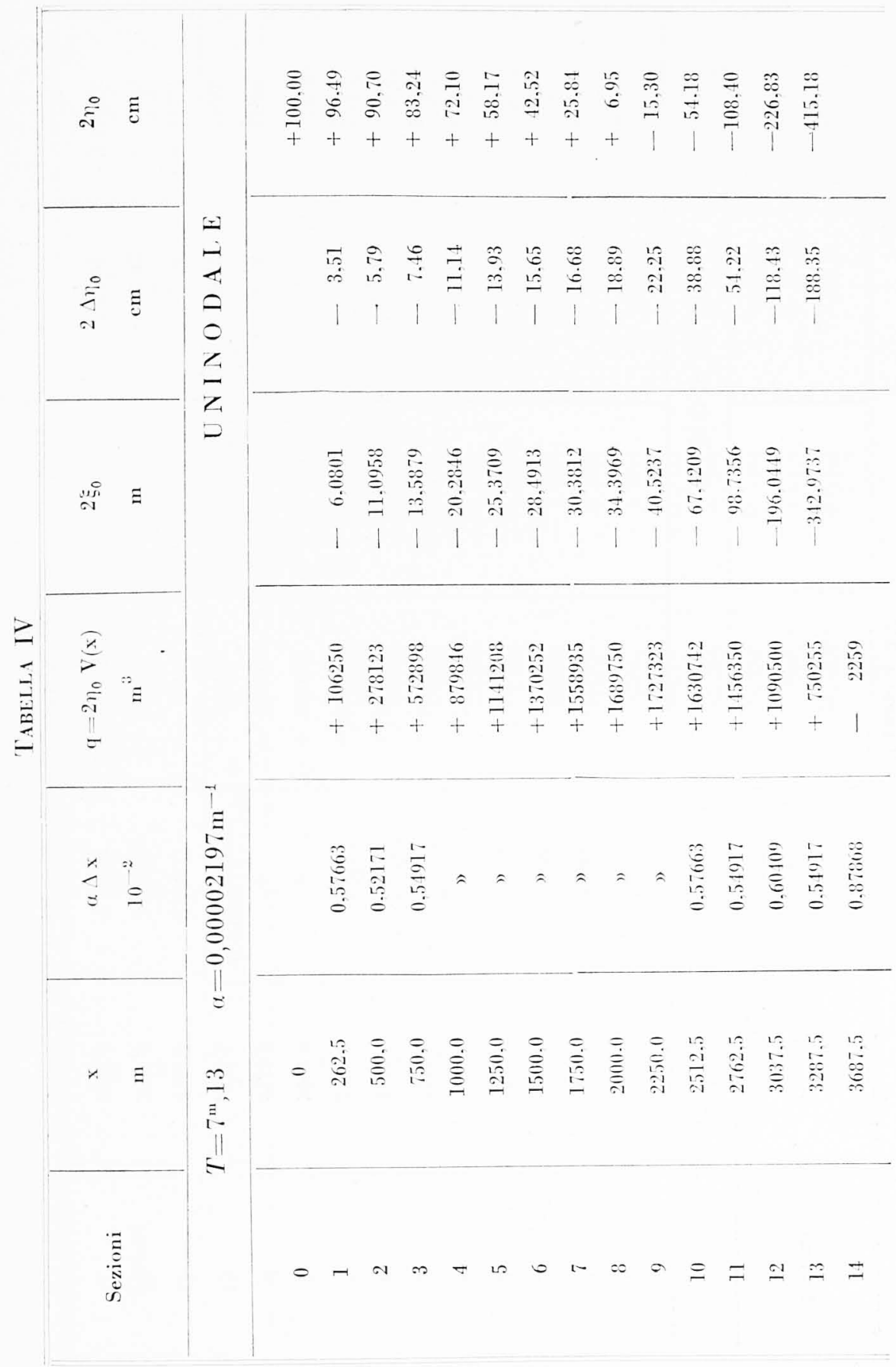




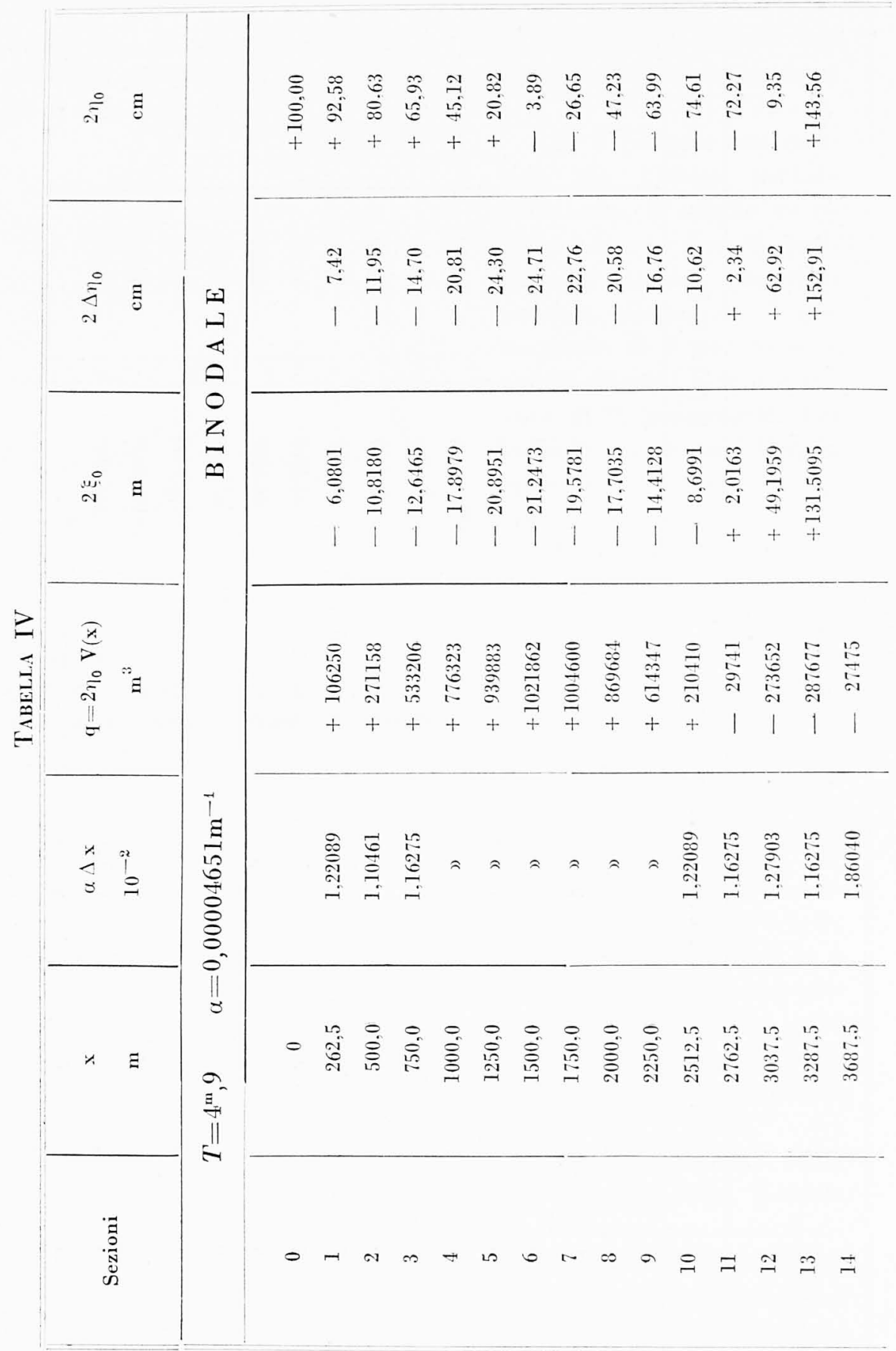




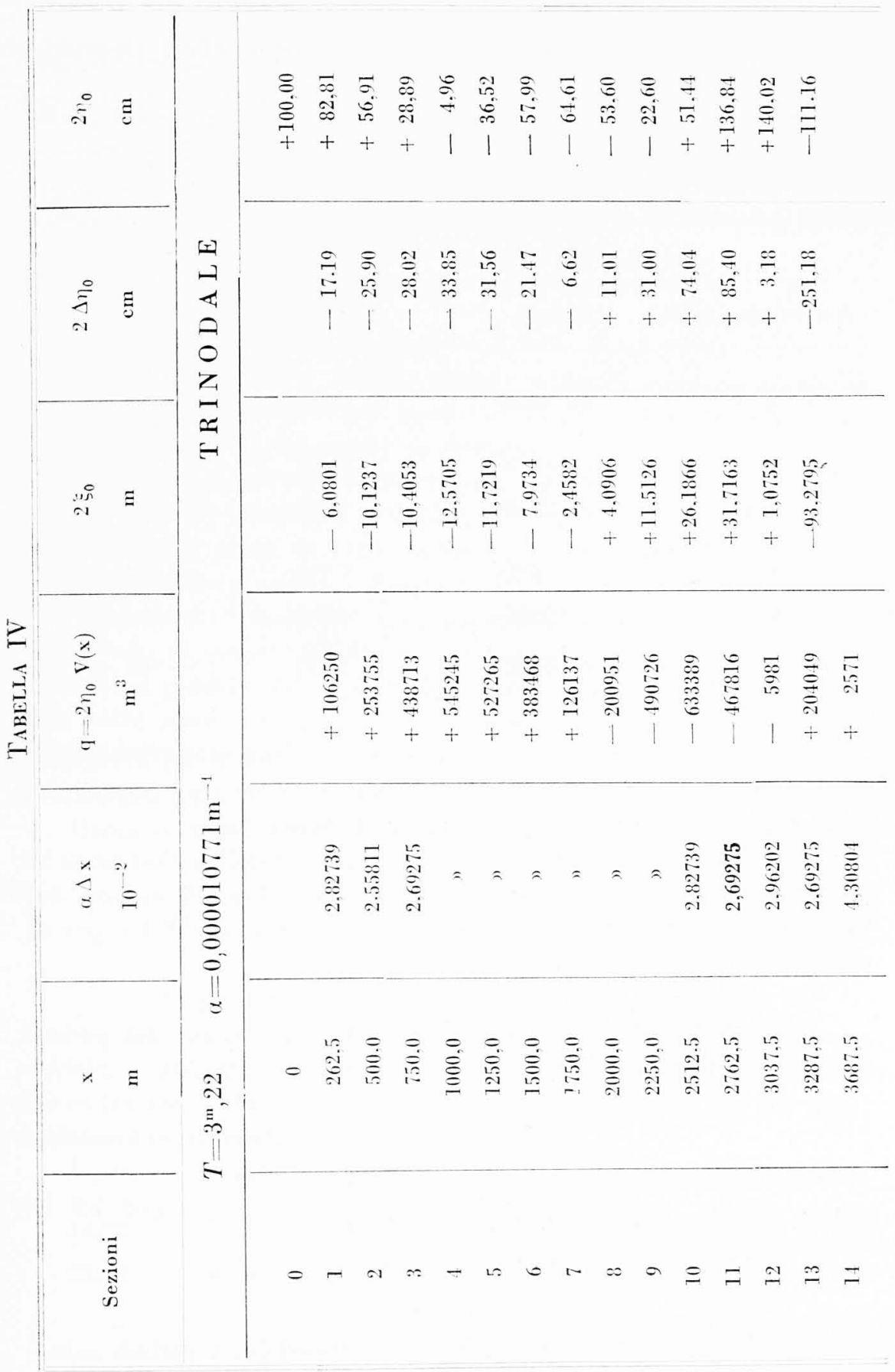


gole sezioni; nella labella IV. colomma 210 -0mo infatti riportati i valori corrispondenti.

Felle figure 2, 3 e then llallo colltinuo sono ripoltali i gralfiri relativi. in confromto con gyli analoghi otfenuti col metodo di Tidakit.

le ascisec delle linee nodali sono in huon accordo con quelle ottemule col metodo di Hidirka, come risulta dal seguente specebiento:

\begin{tabular}{|c|c|c|c|}
\hline Ascisse dei nodi & Midaka & Defant & Differenze \\
\hline $\mathrm{x}_{1}^{\prime}$ & $\mathrm{m} 2364$ & III 2078 & $286 \mathrm{ml}$ \\
\hline $\mathrm{x}^{\prime \prime}{ }_{1}$ & ») 1555 & 1461 & $94 m$ \\
\hline $\mathrm{x}^{\prime \prime}{ }_{2}$ & " 2919 & $m \quad 30533$ & $-134 "$ \\
\hline$x^{\prime \prime \prime}$ & $\Rightarrow \quad 800$ & $\Rightarrow \quad 963$ & $-163 \%$ \\
\hline$x^{\prime \prime \prime}$ & $m \quad 2086$ & y 2282 & $-196 \%$ \\
\hline $\mathrm{x}^{\prime \prime \prime} ;$ & ") 3132 & ) 3176 & $-11 \%$ \\
\hline
\end{tabular}

5. - Le osservazioni. Vei primi griorni di ollobre del J901. Magrini esegui delle oscervazioni ron un limmografo del tipo giappone-e. posto sul lago nelle vicinanze del paese di Santa Croce.

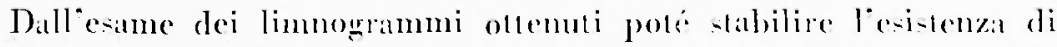
un movimento oscillatorio, specialmente subito dopo il ressatre del rento, con un periodo medio per la sessa uninodale di 7 m. 4 e per la sesá binodale di rilca $5^{m}$.

En esempio di regristazione is riportato nella fig. :.

Xello spereliello seguente somo a ronfronto i valori dei periobi calcolati coi due melodi e quelli osservali:

\begin{tabular}{|c|c|c|c|c|c|c|}
\hline balori & os=errali & & & $\begin{array}{c}\text { unimodale } \\
7^{\mathrm{m}}, 4\end{array}$ & $\begin{array}{l}\text { binodiale } \\
5^{\mathrm{m}}\end{array}$ & Irinodale' \\
\hline ialori & ialcolali & melodo & Miclaka & $7,8.1$ & $\therefore, 7$ & $2 \mathrm{~m}, 61$ \\
\hline ” & ) & ఎ & Defant & 7,13 & 4.9 & 3.22 \\
\hline
\end{tabular}

Si vede che, per le sesse minodale e trinodale, i periodi calcolati con i metodi di Hidaka e di Defant sono leggermente diversi e 
rhe, menter per la prima il melorlo di Tidaka da un valore malggiore di quello di Defiant, per la seconda le cose risulano invertite.

Il ralore osservillo per

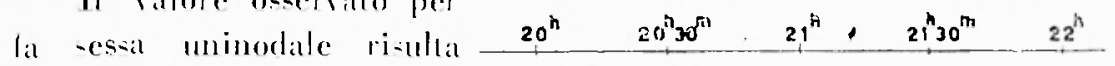
compreso lra i valori calleolali rom i due molocli e no: c quari la media arilnelira. mentre per la hinorlale

5.10.1901. Susa di $i^{m} \cdot 1$

Fig. 5

il valore oservalo risulal di poro -mperiore ai raleolali.

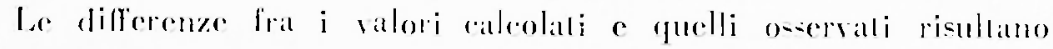
-ufleientemente piecole a per quedle relative ai valori di Dofint minori rhe per guelle dei valori di Hidaka.

Purdrojego il periodo di osecrazione fu mollo limilalo: lullavia i valori ollemuli servono a ronfermare il risultalo dei calcoli coorici dei periodi dei primi dae lipi di sese e dammo indirazione per l'altendibilibi anelar ai valori dei periodi raleolali per la sesea trinodale.

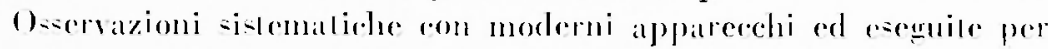
un periodo di lempo suffeiente potrebbero. a-soriate a prove sperimenlali all modello del lateo, sabilire ma media precia dei periodi delle varie sesere equindi rendere possibile un muoro confronto eon i valori ralrolali.

Roma - Istitulo Nazionale di Geofisica - Genmaio 79.18.

\section{RI ISSUNTO}

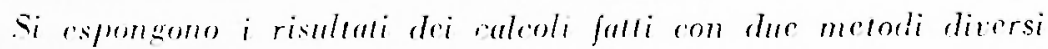
Alidala e Defame! per la deferminazione dei periodi. dei nodi e dellamblamemon delle ampiezen delle sesse dri primi tre hipi del lago di Sama Croos (Bollumo).

$I$ periodi otlenuti risultumo in buon accordo con $i$ ralori osserrali alal Magrini.

\section{BIBLIOGRAFIA}

1) Mackivi G. P.: Contributo allo studio dei laghi hpisini - Memorie Societa geografica italiana 1906. 
Culor P.: Le sesse del lago di Garda - Innali di Goofisica. Parte $1^{20}$ e 2a n. 1 c. 2.1948.

Cunstar, G.: On the hydrodymamical Theory of Serches - Transactions of the Roval Society of Etlimburgh, XLI (1905), XIN (1906), XINI (1907-8).

Huaks K.: Application of Ritz's Tariation Method to the determination of Seiches in a Lake - Mem. Imp. Marine Olserv. VI, n. 2 (1936).

Defaxt A.: Neue Methode zur Ermithlung der Eigenschwingungen (Seiches) von abgeschlossen Whassermassen ecc. - Ann. der Hidrog. XIXI (1918).

Manmix G. P.: Le sesse nei loghi italioni - Rivista geografiea italiana, 1905, 2)ạ. 222. 